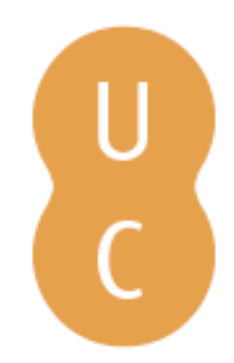

\title{
nombalina
}

\section{Rotas e trilhas dos laboratórios ao conceito de diamagnetismo no século XIX}

Autor(es): $\quad$ Reis, João Batista Alves dos

Publicado por: Imprensa da Universidade de Coimbra

URL

persistente: URI:http://hdl.handle.net/10316.2/32403

DOI: $\quad$ DOI:http://dx.doi.org/10.14195/978-989-26-0469-5_18

Accessed : $\quad$ 26-Apr-2023 14:57:34

A navegação consulta e descarregamento dos títulos inseridos nas Bibliotecas Digitais UC Digitalis, UC Pombalina e UC Impactum, pressupõem a aceitação plena e sem reservas dos Termos e Condições de Uso destas Bibliotecas Digitais, disponíveis em https://digitalis.uc.pt/pt-pt/termos.

Conforme exposto nos referidos Termos e Condições de Uso, o descarregamento de títulos de acesso restrito requer uma licença válida de autorização devendo o utilizador aceder ao(s) documento(s) a partir de um endereço de IP da instituição detentora da supramencionada licença.

Ao utilizador é apenas permitido o descarregamento para uso pessoal, pelo que o emprego do(s) título(s) descarregado(s) para outro fim, designadamente comercial, carece de autorização do respetivo autor ou editor da obra.

Na medida em que todas as obras da UC Digitalis se encontram protegidas pelo Código do Direito de Autor e Direitos Conexos e demais legislação aplicável, toda a cópia, parcial ou total, deste documento, nos casos em que é legalmente admitida, deverá conter ou fazer-se acompanhar por este aviso. 
Ana Leunar Pereira João Rui Pita

[ Coordenaçä̃ ]
Rotas da Natureza

Cientistas

Viagens

Expedifgũes

Instituip̧ües

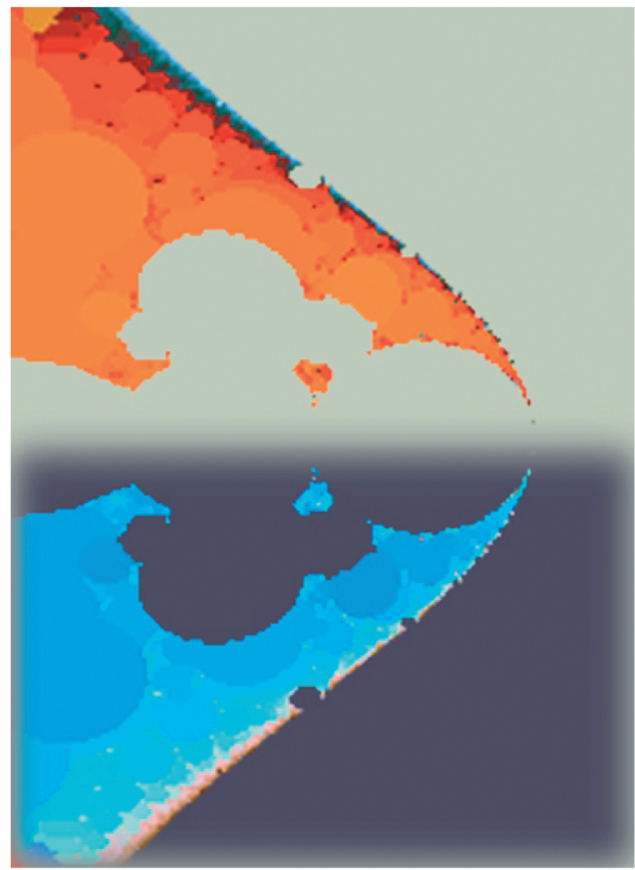




\section{Coordenaçáo Científica da Colecção Ciências e Culturas}

João Rui Pita e Ana Leonor Pereira

Os originais enviados são sujeitos a apreciação científica por referees

\section{Coordenação Editorial}

Maria João Padez Ferreira de Castro

\section{Edição}

Imprensa da Universidade de Coimbra

Email:impresauc@ci.uc.pt

URL: http://www.imp.uc.pt • Normas de publicação de colecçôes

\section{Design}

António Barros

Pré-Impressáo

António Resende

Imprensa da Universidade de Coimbra

\section{Capa}

António Barros, com imagem de E. M. de Melo e Castro, 2003 [Fractal original gerado no Fractint com tratamento no Photoshop 7.0]; Cortesia: António Barros

Impressão e Acabamento

SerSilito • Maia

\section{ISBN}

978-989-8074-12-6

\section{Depósito Legal}

Obra publicada com a colaboraçáo de:
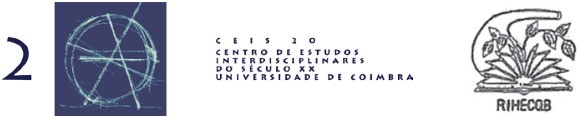

Obra publicada com o apoio de:

FCT Fundação para a Ciência e a Tecnologia

MINISTÉRIO DA CIÊNCIA, TECNOLOGIA E ENSINO SUPERTOR Portug

Programa Operacional Ciência, Tecnologia, Inovação do Quadro Comunitário de Apoio III
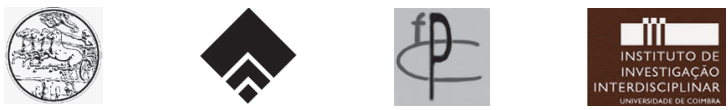

\section{Baxter}

(c) 2006, Imprensa da Universidade de Coimbra 
João Rui Pita

Ana Leonar Pereira

(Courdenação)

Rotas da Natureza

Cientistas

Viagens

Expediçũes

Instituiç̃̃es

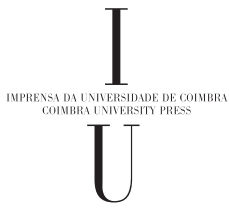

- colmbra 2006 
(Página deixada propositadamente em branco) 
João Batista Alves dos Reis

Centro Universitário de Caratinga - UNEC - Caratinga - Minas Gerais, Brasil

\section{ROTAS E TRILHAS DOS LABORATÓRIOS AO CONCEITO DE DIAMAGNETISMO NO SÉCULO XIX}

\section{Introdução}

«As anomalias de polaridade» em cristais de bismuto foram observadas por Anthony Brugmans, nos idos de 1778, tornam-se por vários anos do século XIX na Royal Institution, o foco principal de estudos e experimentos sobre polaridade paramagnética e diamagnética. ${ }^{11}$

Para Michael Faraday (1791-1867), estudioso britânico, essas anomalias, eram deflexões reversas de polaridade geradas por disposição anômala nas linhas de força magnéticas. Processo semelhante à repulsão ocorrida entre pólos iguais de magnetos comuns. Denominou esse comportamento, a princípio de diamagnetismo, pois apesar das linhas de força fluírem sobre esses corpos, essa ação, não definia o estado magnético usual do ferro ou da pedra-ímã (loadstone).

O estudioso britânico, em 2 de Fevereiro de 1846, adicionou na série XXI de 22 de Dezembro de 1845 do Experimental Researches in Electricity (ERE), que as observações da ação de magnetos sobre metais e seus compostos haviam sido relatadas, desde 1778, por Anthony Brugmans - Antonii Brugmans Magnetismus seu de affinitatibus magneticis observationes magneticae. Lugd. Batav. 1778, parágrafo 41.

Segue na mesma nota referências de publicações: no Bulletin Universel de 1827 e de 1828, citando os ensaios de M. de le Baillif «Repulsion of a Magnet by Bismuth and Antimony" [1827], de Saigey "Magnetism of certain natural combinations of Iron and on the mutual repulsions of Bodies in general», e ainda os trabalhos teóricos de Seebeck «Magnetic Polarity of different Metals, Alloys and Oxides»

\footnotetext{
${ }^{1}$ Entre 1845 a 1850, Michael Faraday concluía um conceito de extraordinária relevância como ferramenta teórica sobre a estrutura da matéria nas interfaces conceituais entre física e química. Normalmente, uma larga classe de substâncias avaliadas experimentalmente com uma taxa muito pequena de susceptibilidade magnética, foi denominada de substância diamagnética. Em cada um desses materiais, a direcção da magnetização era oposta à direcção do campo induzido. As substâncias paramagnéticas caracterizam-se por seus domínios magnéticos serem atraídos do mesmo modo que os ferromagnéticos, mas com intensidade mais fraca.
} 
Ainda conforme Michael Faraday, o efeito das «anomalias de polaridade» em gases e chamas de gases, foi observado pela primeira vez por P. Bancalari e, retratado num artigo publicado pelo professor Zantedeschi, em 1847, na Itália. A pedido de Faraday em carta enviada ao Esquire Richard Taylor, então editor, foi publicado também na Inglaterra no Philosophical Magazine.

Distintos conceitos, a priori, seriam discutidos por vários contemporâneos de Faraday nesse assunto, bem como intensos debates surgiriam das pressuposições aqui citadas pelo estudioso britânico em relação à divisão do sistema magnético em substâncias para e diamagnéticas.

Para o estudioso britânico, estava sendo definida uma "nova condição magnética», ou melhor, uma «nova força magnética» ou ainda uma «ação da matéria diamagnética». Todavia, foi a partir dos experimentos sobre a polarização da luz envolvendo, principalmente, cristais diamagnéticos e gases diamagnéticos, que Michael Faraday estruturou afinal a sua teoria sobre a matéria diamagnética conjugando-a à indução eletromagnética.

Portanto, a busca de um modelo de unificação das forças da natureza, parece ter norteado os estudos de Faraday sobre o diamagnetismo, como uma unidade padrão de convertibilidade da matéria magnética.

As estruturas teórico-experimentais analisadas e relatadas no ERE e no Diário de Laboratório de Faraday, sugerem, que a teoria fundamental do magnetismo estava fundamentada em quatro pilares cujos argumentos básicos eram; a condição da condução nas linhas de força um conceito relacionado às idéias de campo, a convertibilidade pela indução o magnetismo converte-se em eletricidade, a convertibilidade da matéria magnética relacionada às altas temperaturas, teoricamente todos os materiais nessas condições convertem-se em «um estado diamagnético».

\section{Os experimentos com cristais diamagnéticos: uma definição da rota conceptual do diamagnetiemo}

Michael Faraday descreveu, após vários experimentos com diferentes substâncias paramagnéticas e diamagnéticas, que o torque ou momento da força magnética agia sobre essas substâncias através de dois estágios de equilíbrio. Esses, dependiam dos arranjos da estrutura química em questão, ou seja, a rotação em alguns desses corpos era diferente entre os intervalos de instabilidade - no inicio do experimento - e de estabilidade no decorrer do experimento.

O efeito da torção do momento se dava a partir de uma força que causava várias rotaçōes, um efeito interessante, pois ocorria diferentemente para cada corpo em razão da estrutura molecular. Essas especificidades, dos efeitos de torção, foram observadas no antimônio, no estanho, no bismuto e, em várias espécies de metais e vidros metálicos. O efeito observado nessas substâncias, foi executado tendo em vista o uso de uma mesma intensidade padrão de força magnética ordinária para cada experimento.

Citando o eminente pesquisador da Royal Institution:

[...] Estimulado por tais sentimentos, estive engajado com Plücker, Weber, Reich e outros, no empenho de compreender, com algum grau de precisão, o modo da ação 
de um diamagnético bem como dos corpos «magnetocristálicos»; [...] e no empenho para confirmar a idéia de polaridade do bismuto e dos corpos diamagnéticos, a [direção] reversa [tomada] por eles em [relação] aos magnetos, [...] foi um dos primeiros resultados dessa convicção e desejo. [...]. ${ }^{2}$

Em novembro de 1847, Plücker ainda informaria a Faraday, que havia descoberto que os pólos magnéticos eram centros de uma nova força interagindo com os eixos ópticos dos cristais e que essa nova força diminuía mais rapidamente com a distância do que pela intensidade menor das forças tanto magnéticas quanto diamagnéticas.

Plücker tentava demonstrar que um corpo poderia comportar-se ao mesmo tempo como magnético ou diamagnético, dependendo apenas da distância em relação ao magneto. No entanto, para Faraday o comportamento dos diamagnéticos era extremamente discreto e "mascarado" por resíduos magnéticos das linhas de força, exibindo açōes e efeitos indesejáveis. Argumentava que tais aspectos eram de ordem estrutural que:

[...] um corpo magnético tende dos lugares de ação magnética mais fraca para os de forte ação e um corpo diamagnético sob as mesmas condições dos lugares de ação magnética forte para lugares de fraca ação. [...].3

A luz polarizada comportava-se quanto à condutividade de forma muito parecida com a observada nos corpos diamagnéticos, ou seja, concentrava-se também numa posição de menor energia no campo magnético. Tanto a luz como o efeito diamagnético seriam "poderes», todavia sutis peculiaridades os diferenciavam.

A rotação do raio de luz mudaria somente se a polaridade do eletromagneto fosse mudada e, nesse caso, o poder da rotação da luz polarizada seria diretamente proporcional à intensidade da força magnética.

Faraday argumentava que não era a polaridade dos magnetos nem a ação dos dielétricos, e sim as linhas de força e as diversas peculiaridades do raio de luz, que definiam os principais aspectos desse efeito.

[...] Minha visão de polaridade está fundamentada na característica tomada por ela mesma em direção às forças, [...] quando um condutor elétrico se move em uma mesma direção perto ou entre corpos age magneticamente sobre si mesmo ou entre eles, há uma corrente constante produzida, a polaridade magnética é a mesma; se o movimento ou a corrente forem invertidas, a polaridade magnética é indicada. A indicação é na verdade ou para o exterior ou para o interior dos corpos magnéticos sempre que a corrente é produzida, e depende do desconhecido mas essencial caráter dual ou antitético natural das forças a qual chamamos magnetismo.[...]. ${ }^{4}$

\footnotetext{
${ }^{2}$ Michael Fraday, Experimental Researches in Electricity, p. 523, vide também, página 538, parágrafo 2310 .

${ }^{3}$ Ibid., p. 594, parágrafo 2789.

${ }^{4}$ Ibid., p. 740-741, parágrafos 3307, 3308 e 3309.
} 
Tais aspectos complementavam as afirmaçôes anteriores de Faraday sobre a questão dos corpos magnéticos, quando submetidos a grandes mudanças de temperatura, reterem sua porção magnética numa espécie de «estado diamagnético»:

[...] Os metais que são magnéticos retêm uma porção de seu poder após grandes mudanças de temperatura, sendo afetados no que poderíamos chamar de «seu estado diamagnético»; mas os outros metais, tais como bismuto, estanho, \&c., não apresentam sequer traços desse poder, no entanto não estavam na condição de aquecimento que o ferro, níquel ou cobalto [...] durante a mesma situação eles são atraídos axialmente e noutras repelidos equatorialmente. [...]. ${ }^{5}$

Assim, para o eminente pesquisador britânico, as forças elétrica e magnética não só exerceriam forças de polarização, como também de despolarização da luz. Indubitavelmente, esses aspectos, continham uma influência especial que deveria ocorrer também sobre as demais formas de forças, tais como: nos agentes radiantes, o calor, e a força química. ${ }^{6}$

\section{Conclusão}

Ainda, no século XIX, deve-se salientar os trabalhos de Emil Lenz, Williams Thomson, Wilhelm Weber, Julius Plücker, Henry Becquerel e de John Tyndall. Entretanto, foram a partir das mensuraçôes quantitativas de susceptibilidade magnética nos cristais paramagnéticos. Assim, o efeito diamagnético retorna ao cenário de novos estudos.

A idéia do diamagnetismo, no século XX, foi desenvolvida através dos trabalhos de Paul Langevin, principalmente, os oriundos do conceito de proporcionalidade da ação diamagnética nos campos moleculares serem intrínsecos à magnetização, confirmada por Pierre Weiss (1907).

Em 1920, Wolfgang Pauli obtinha através da fórmula de Langevin um valor negativo para a susceptibilidade magnética, estudando o diamagnetismo das substâncias ionizadas.

O «efeito Zeeman normal» de Pieter Zeeman observado experimentalmente por John Hasbrouch van Vleck, em relação à susceptibilidade magnética molecular, era definido pelo gradiente menor ou maior do estado de energia, concernente, ao momento magnético orbital obtido nos estados fundamental e excitado.

Confirmava-se, então, a influência da condição molecular e convertibilidade do momento magnético, do grau de susceptibilidade magnética para conceituar os corpos diamagnéticos, conforme Faraday já havia predito em suas obras publicadas no século XIX.

\footnotetext{
5 Ibid., p. 528 parágrafo 2348.

6 Ibid., p. 514
} 
Em 1920, através das medidas residuais das substâncias diamagnéticas ionizadas, Wolgang Pauli determinou uma unidade fundamental do momento magnético de um elétron.

Em 1933, Walther Meissner e R. Oschenfeld observam que supercondutores expulsavam todo o fluxo magnético do seu interior, ou seja, os supercondutores se comportavam como um material perfeitamente diamagnético. Divulga-se que os corpos diamagnéticos tinham características semelhantes ao comportamento dos materiais supercondutores.

Durante a segunda metade do século XX, as pesquisas magnéticas, paramagnéticas e diamagnéticas, expandir-se-iam à modelagem ao uso de sensores de dipolos de correntes equivalentes para a despolarização dos órgãos biológicos monitorados, gerando campos magnéticos.

O uso de medidores de susceptibilidade magnética para quantificar substâncias ferromagnéticas ou diamagnéticas, por exemplo, a quantidade de átomos de ferro contido no sangue, essa medida, proporciona investigar os campos magnéticos gerados pelo próprio organismo ou os estímulos de ações externas.

Assim como, a captação e a medição de campos magnéticos e ruídos nas faixas de amplitudes e freqüências baixas. Configuram-se imagens bem nítidas das características anatômicas de órgãos biológicos principalmente as particularidades essenciais à preservação da vida.

Órgãos tais como, o coração (adulto e fetal), por exemplo, pela despolarização do átrio a partir do nódulo sino-atrial, bem como o trato gastrointestinal, o cérebro (adulto e fetal), o fígado, e o pulmão. Atualmente, existem mais de 50 grupos de pesquisa em biomagnetismo, sendo quatro deles no Brasil.

\section{REFERÊNCIAS BIBLIOGRÁFICAS}

BERKSON, W. Las teorías de los campos de fuerzas desde Faraday hasta Einstein. Madrid, Alianza Universidad, 1981.

CANTOR, Geoffrey. «How Michael Faraday Brought Law and Order to the West End of 7 London». Physis XXIX: 187-203, 1992.

Caroe, Gwendy. The Royal Institution : An Informal History. London, John Murray Publishers, 1985.

DORAN, Barbara Gusti. "Field Theory in $19^{\text {th }}$ Century Britain" : in Historical Studies in the Physical Sciences. New Jersey, Princeton University, 1975.

FARADAY, Michael. Experimental Researches in Electricity. Chicago/London, (Great Books of

Western World). Vol. 42, Enciclopædia Britannica, 1994.

—. The Forces of Matter. Buffalo. (Great Minds Series), Prometheus Books, 1993.

Fisher, H. J. «Faraday Two Voices». Physis XXIX : 166-186, 1992.

Gooding, David. "Mathematics and Method in Faraday’s Experiments. Physis XXIX : 121-147, 1992.

—.«Empiricism in Practice : Teleology, Economy, and Observation in Faraday's Physics». ISIS : 46-67, 1982.

Hare, E. H. «Michael Faraday’s Loss of Memory». Proc. Soc. Med. 67: 617-618, 1974.

Hoffmann, Banesh. Relativity and its Roots. Mineola/New York, Dover, 1999. 
Knight, David M. The Age of Science : The Scientific World View in Nineteeth Century. Oxford/New York, Basil Blackwell, 1986.

NerSessian, Nancy J. Faraday to Einstein : Constructing Meaning in Scientific Theories. London, Kluwer Academic Publishers, 1984.

OlDROYD, David Roger. The Arch of Knowledge : An Introductory Study of the History of the Philosophy and Methodology of Science. New York/London, Methuen, 1986.

TwENEY, Ryan D. «Mathematical Thinking about Experimental Matters: Faraday as a Mathematical Philosopher». In : Rediscovering Skill in Science, Tecnology and Medicine, Science Studies, 1990.

—. «Stopping Time : Faraday and the Scientific Creation of Perceptual Order», PHYSIS: 149-164, 1992.

William, L. Pearce. «Faraday, Michael». In : Charles Coulston Gillispie (org). Dictionary of Scientific Biography. Vol 4. New York, Charles Scribner's, 1980, pp. 527-539. 
(Página deixada propositadamente em branco) 


\section{Colecçãa \\ 2 Ciências e Culturas Caimbra 2006}

